\title{
High prevalence of cisplatin-induced ototoxicity in Cape Town, South Africa
}

\author{
H Whitehorn, ${ }^{1}$ BSc, BSc (Med) Hons, MSc (Med); M Sibanda, ${ }^{2}$ BSc, MSc (Audiology); M Lacerda, ${ }^{3}$ BBusSc Hons, MSc, PhD; \\ T Spracklen, ${ }^{1}$ BSc, BSc (Med) Hons; L Ramma, ${ }^{2}$ AuD, MPH, CCC-A; S Dalvie, ${ }^{4}$ MB ChB, FC (Rad Oncol); \\ R Ramesar, ${ }^{1}$ MSc (Microbiology, Biochemistry, Genetics), PhD (Microbial Genetics), PhD (Molecular Genetics), MBA \\ ${ }^{1}$ Division of Human Genetics, Department of Clinical Laboratory Sciences, Faculty of Health Sciences, University of Cape Town, South Africa \\ ${ }^{2}$ Division of Communication Sciences and Disorders, Groote Schuur Hospital, Cape Town, South Africa \\ ${ }^{3}$ Department of Statistical Sciences, Faculty of Science, University of Cape Town, South Africa \\ ${ }^{4}$ Department of Radiation Oncology, Groote Schuur Hospital, Cape Town, South Africa
}

Correspondingauthor: R Ramesar (raj.ramesar@uct.ac.za)

\begin{abstract}
Background. Cisplatin is administered as the first-line treatment of soft-tissue cancers. It has a reported cure rate of up to $85 \%$, but is associated with a high incidence of ototoxicity, characterised by irreversible bilateral hearing loss and affecting $23-50 \%$ of adults who receive the drug. Objectives. To determine the incidence of cisplatin-induced ototoxicity at Groote Schuur Hospital (GSH), Cape Town, South Africa. Methods. A retrospective cross-sectional study of cisplatin-receiving cancer patients attending GSH between January 2006 and August 2011. Results. A total of 377 patients were recorded as receiving cisplatin therapy during the study period. A $300 \%$ increase in new cisplatin-receiving patients receiving audiological monitoring was observed between 2006 and 2010 . However, only patients with all clinical data as well as baseline and follow-up audiometric analyses were investigated. One hundred and seven such patients were identified, $55.1 \%$ of whom developed cisplatininduced ototoxicity while receiving high-dose $\left(\geq 60 \mathrm{mg} / \mathrm{m}^{2}\right)$ cisplatin treatment. Higher cumulative cisplatin dosages were associated with development of significant hearing loss $(p=0.027)$. The odds of developing cisplatin-induced hearing loss were elevated for patients with head and neck tumours and lymphoma ( $p=0.0465$ and $p=0.0563$, respectively) and were significantly lower for those with reproductive cancers ( $p=0.0371$ ). Conclusion. Comprehensive audiological monitoring should be available for every patient during cisplatin treatment to minimise the development of disabling hearing loss.
\end{abstract}

S Afr Med J 2014;104(4):288-291. DOI:10.7196/SAMJ.7389

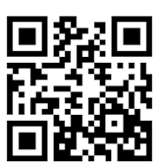

Cisplatin is one of the most widely used and effective cytotoxic agents currently available for the treatment of soft-tissue cancers, with a reported cure rate of $85 \%{ }^{[1]}$ This antineoplastic drug inhibits tumour growth through the formation of DNA interstrand and intrastrand crosslinks, which disrupts the double-stranded DNA helical structure. This in turn prevents cell division and growth due to inhibition of DNA replication and transcription, leading directly to apoptosis. ${ }^{[2]}$

Use of cisplatin is limited by the relatively high incidence of associated adverse drug reactions, including neurotoxicity, ototoxicity and nephrotoxicity, due to the drug's narrow therapeutic index. Cisplatininduced ototoxicity is characterised by high-frequency sensorineural bilateral hearing loss, which can be irreversible and progressive ${ }^{[3]}$ and is often associated with tinnitus and ear pain. The hearing loss usually begins at high frequencies $(>8000 \mathrm{~Hz})$ and may later progress to lower frequencies that are important for conversational speech.

According to international reports, cisplatin ototoxicity affects $23-50 \%$ of adults and up to $60 \%$ of children. ${ }^{[4-6]}$ However, some studies have reported elevated hearing thresholds in up to $100 \%$ of cisplatin-treated cancer patients. ${ }^{[1]}$ Limited data are available on the incidence of drug-induced hearing impairment in Africa. The reported prevalence of cisplatin-induced hearing impairment and tinnitus is strongly dependent on the criteria and diligence of the audiological evaluation. The Chang ototoxicity grading scale is a modification of the original Brock criteria for ototoxic hearing loss. ${ }^{[7]}$ Importantly, these criteria specify the severity of hearing loss and can be used reliably by audiologists, oncologists and clinical research personnel to standardise ototoxicity monitoring.
Drug-induced ototoxicity negatively influences an individual's verbalauditory communication, resulting in a detrimental effect on learning and socio-emotional status. ${ }^{[5]}$ Hearing loss can have educational and economic disadvantages including delayed speech acquisition and literacy and impaired cognitive skills in children, and social isolation. These consequences are greater in developing countries because of the inadequacy of rehabilitation and social service facilities. The development of adverse drug reactions such as ototoxicity often leads to discontinuation of cisplatin treatment or reduction of the dose. This affects compliance with treatment and ultimately leads to decreased survival rates in cancer patients.

Variation in susceptibility to the development of cisplatin-induced ototoxicity has been attributed to age at initiation of treatment, renal dysfunction, cumulative cisplatin dosages, cranial irradiation, co-administration with other ototoxic drugs, noise exposure and genetic factors. ${ }^{[8-10]}$ The majority of studies investigating cisplatininduced ototoxicity have been performed on European and South American patient cohorts, ${ }^{[4-6,10]}$ with only a single study examining the development of ototoxicity in Japanese head and neck cancer patients. The incidence rate of cisplatin-induced ototoxicity was reported as $77.3 \%$ in the 44 Japanese patients investigated. ${ }^{[11]}$

No clinically proven treatments for the prevention or amelioration of ototoxicity exist, although recent studies have highlighted the value of co-administration of cisplatin with otoprotectors such as D-methionine. ${ }^{[12]}$ However, the development of ototoxicity remains the most significant dose-limiting factor in cisplatin-based chemotherapy owing to the cumulative, and often irreversible, manner in which it develops. Ototoxicity frequently affects the outcome of treatment, 
yet its prevalence in cancer patients in South Africa (SA) is largely unreported. This study aimed to determine the incidence of cisplatin-induced ototoxicity in a population of patients attending Groote Schuur Hospital (GSH), Cape Town, SA.

\section{Methods \\ Patients}

A 5-year retrospective cross-sectional study was carried out on patients receiving highdose ( $\geq 60 \mathrm{mg} / \mathrm{m}^{2}$ per cycle) cisplatin-based chemotherapy with audiological monitoring at GSH between January 2006 and August 2011. Ethical approval was granted by the Human Research Ethics Committee, University of Cape Town.

Socio-demographic and clinical data including pre-existing hearing loss, previous noise exposure, anatomical site of cancer and cumulative cisplatin dosage $\left(\mathrm{mg} / \mathrm{m}^{2}\right)$ were collected from patient medical records. Inclusion criteria were the availability of a baseline, and at least one additional, audiological assessment.

\section{Audiometric monitoring}

Assessment of hearing thresholds was conducted using conventional pure-tone audiometry in an audiometric booth using a GSI 61 audiometer. Air and bone conduction thresholds were determined at frequencies between 0.5 and $8 \mathrm{kHz}$. Clinical ototoxicity was classified according to the American Speech-Language-Hearing Association (ASHA) guidelines ${ }^{[13]}$ as a $20 \mathrm{~dB}$ HL or greater hearing loss decrease in pure-tone threshold at any single test frequency or a $10 \mathrm{~dB} \mathrm{HL}$ or greater hearing loss decrease at any two adjacent test frequencies in air conduction. Patients presenting with significant hearing loss following cisplatin treatment were further classified according to severity using the Chang ototoxicity scale. ${ }^{[7]}$

\section{Statistical analysis}

Descriptive statistics were used to analyse the data using the statistical environment $R$ and the IBM SPSS statistics package. KaplanMeier survival analysis was used to assess the rate of development of ototoxicity based on the time to hearing loss after initiation of cisplatin treatment. $p$-values of $<0.05$ were considered statistically significant.

\section{Results}

A total of 377 patients received cisplatin therapy during the study period. One hundred and seven cisplatin-receiving patients with comprehensive clinical and audiometric data were identified; however, clear cumulative cisplatin dosages were only available for 86

Table 1. Demographic characteristics of individuals with and without ototoxicity following cisplatin treatment

\begin{tabular}{|c|c|c|c|}
\hline & $\begin{array}{l}\text { Ototoxicity free } \\
(N=48)\end{array}$ & $\begin{array}{l}\text { Ototoxicity } \\
(N=59)\end{array}$ & $p$-value \\
\hline Age (years), mean (min; max) & $41.6(14 ; 72)$ & $44.4(14 ; 75)$ & $0.910^{*}$ \\
\hline Gender (male), $n(\%)$ & $37(77.1)$ & $42(71.2)$ & $0.665^{\dagger}$ \\
\hline $\begin{array}{l}\text { Cumulative cisplatin dose }\left(\mathrm{mg} / \mathrm{m}^{2}\right) \text {, median } \\
(\mathrm{min} ; \max )\end{array}$ & $\begin{array}{l}180.70 \\
(79.11 ; 431.65)\end{array}$ & $\begin{array}{l}236.84 \\
(47.62 ; 511.56)\end{array}$ & $0.027^{\star \ddagger}$ \\
\hline \multicolumn{4}{|c|}{$\begin{array}{l}\text { Ototoxicity free = patients who did not develop significant hearing loss; ototoxicity = patients who developed significant } \\
\text { hearing loss. } \\
\text { 'Wilcoxon rank-sum test. } \\
\text { 'Fisher's exact test. } \\
\text { FStatistically significant at type } 1 \text { error rate of } 5 \% \text {. }\end{array}$} \\
\hline
\end{tabular}

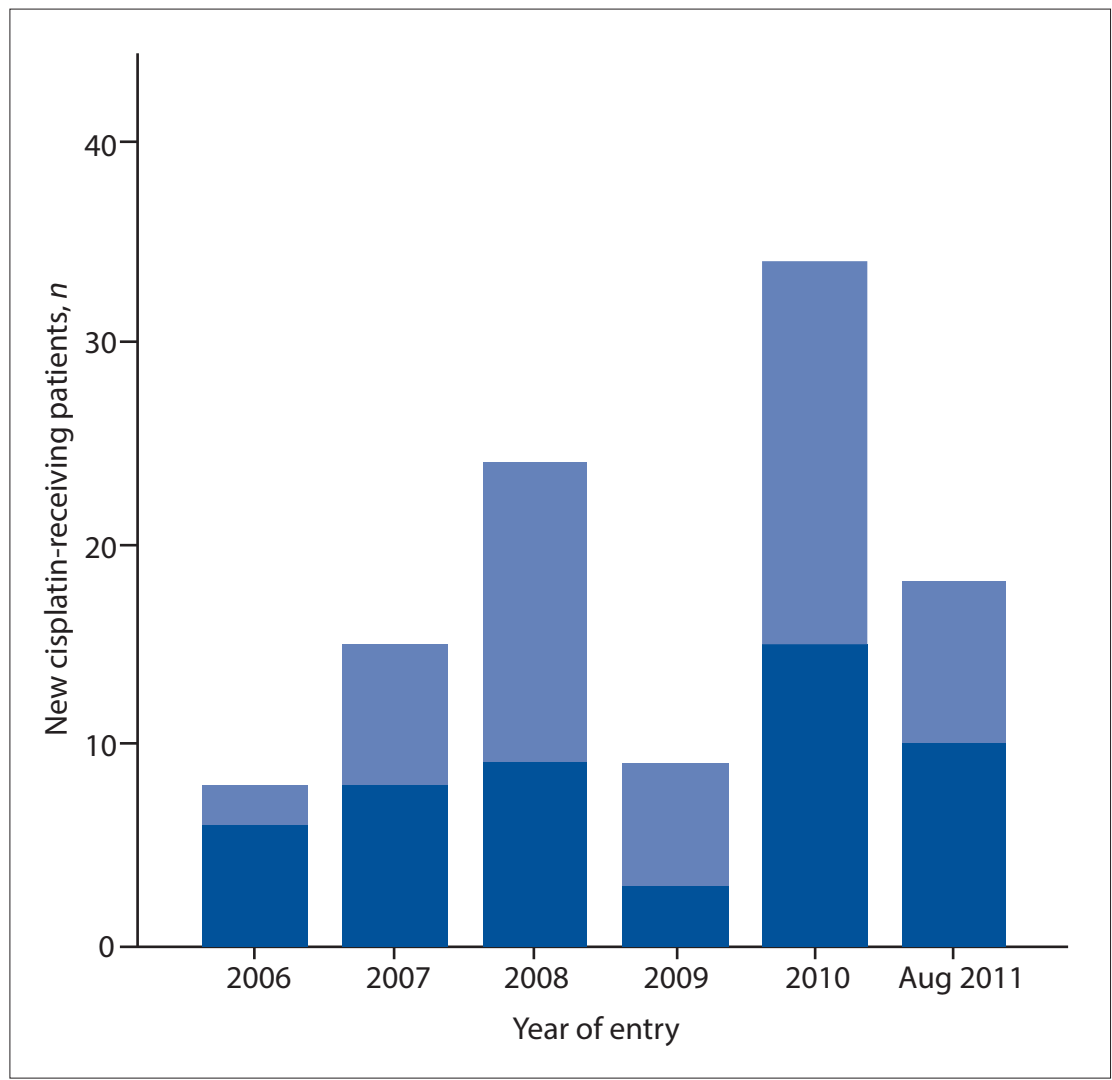

Fig. 1. Frequency of new cisplatin-receiving patients with audiological monitoring at Groote Schuur Hospital. The frequency of these patients displays an increasing exponential trend between 2006 and 2010. Pale blue represents patients who experienced significant cisplatin-induced hearing loss and dark blue represents patients who did not experience hearing loss.

of these patients. The study cohort consisted of 79 males $(73.8 \%)$ and 28 females $(26.2 \%)$. Patients were classified into two groups, based on the development of significant hearing loss following cisplatin-containing chemotherapy the ototoxicity-free (no significant hearing loss) and ototoxicity (significant hearing loss) groups.

The ototoxicity-free group comprised 48 patients, of whom $77.1 \%$ were male, and the ototoxicity group 59 patients, of whom $71.2 \%$ were male (Table 1). The mean ages for the ototoxicity-free and ototoxicity groups were 41.6 and 44.4 years (range 14 - 72 years and 14 - 75 years), respectively. Gender was not associated with significant hearing loss (Fisher's exact test, $p=0.665$ ), and there was no significant difference in the age distribution for patients who did and did not experience hearing loss (Wilcoxon test, $p=0.910$ ). A total incidence of cisplatin-induced ototoxicity of $55.1 \%$ was observed in this SA population, of whom $62.7 \%$ experienced bilateral hearing loss. Only 3 patients in the ototoxicity cohort had self-reported previous noise exposure, and another 2 reported experiencing tinnitus during cisplatin treatment. 


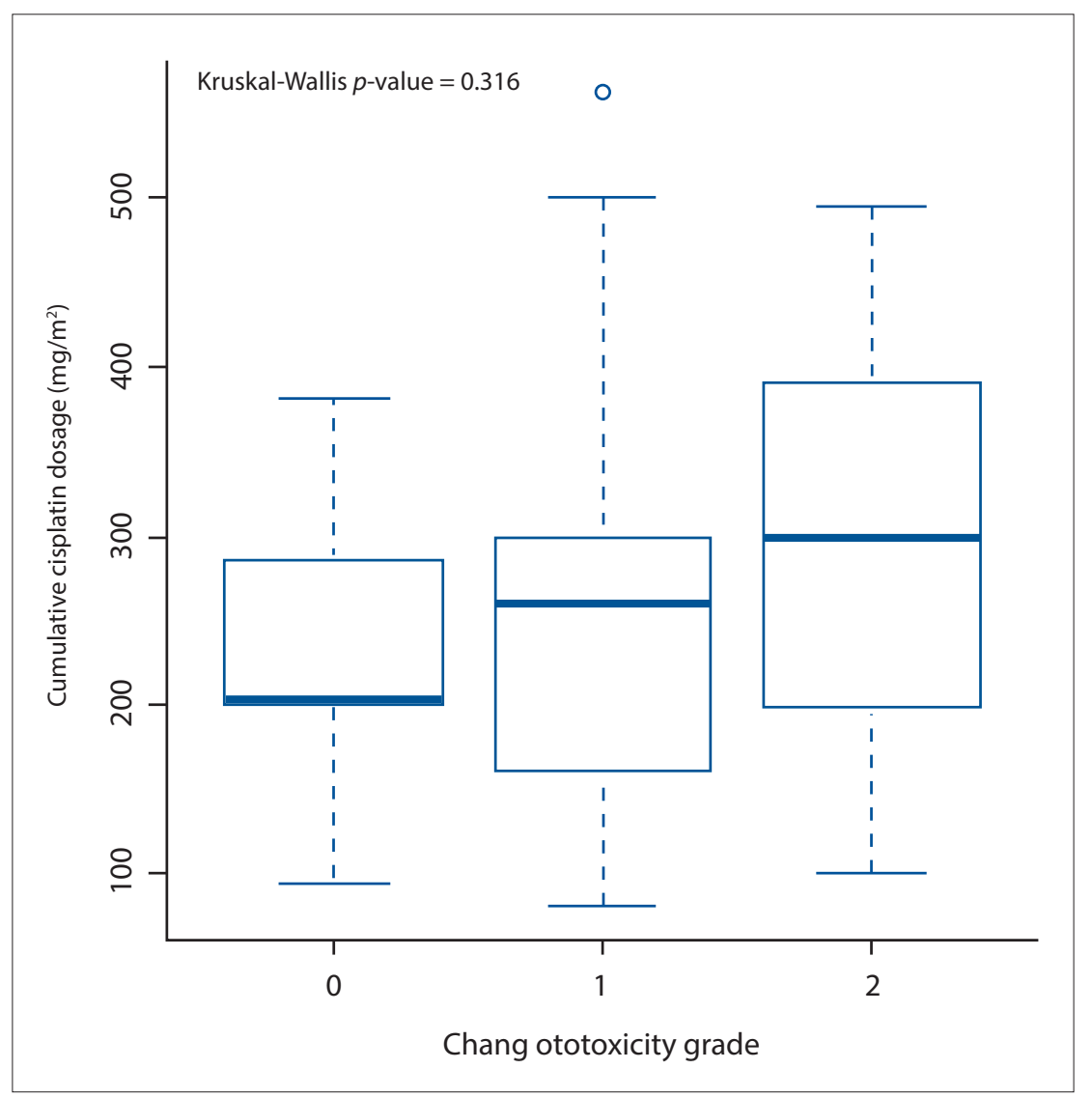

Fig. 2. Distribution of cumulative cisplatin dosage based on severity of hearing loss. The distribution displays an increasing trend with increasing severity of hearing loss experienced. The Chang ototoxicity grading scale was used to assess severity, with grades $1 A$ and $1 B$ and $2 A$ and $2 B$ grouped into grades 1 and 2, respectively, owing to small sample size. Patients with grade 3 ototoxicity $(\mathrm{n}=3)$ are not shown.

Approximately half of the study participants (53.3\%) had hearing loss before initiation of cisplatin-based chemotherapy, as classified by ASHA. ${ }^{[13]}$ As expected, pre-existing hearing loss was significantly associated with age at baseline (Wilcoxon test, $p<0.001$ ), but it was not associated with subsequent cisplatin-induced hearing loss (Fisher's exact test, $p=0.696$ ). The age distribution differed significantly according to anatomical tumour location $(p<0.001)$, patients with reproductive cancers and osteosarcoma being younger at baseline audiometric analysis.

The frequency of new cisplatin-receiving patients per year, who were audiologically monitored during treatment, increased by $300 \%$ between years 2006 and 2010, with the exception of 2009 (Fig. 1). It is expected that the frequency of cisplatin-receiving patients continued to increase between September and December 2011, since data were only recorded up to the end of August 2011.

The median cumulative cisplatin dosages were $180.70 \mathrm{mg} / \mathrm{m}^{2}$ and $236.84 \mathrm{mg} / \mathrm{m}^{2}$ for the ototoxicity-free and ototoxicity groups, respectively. Patients who received higher cumulative dosages during chemotherapy were more likely to develop significant hearing loss (Wilcoxon test, $p=0.027$ ) (Table 1). The median cumulative cisplatin dosages displayed an increasing trend with the severity of the hearing loss, although this association was not statistically significant (Kruskal-Wallis test, $p=0.316$ ) (Fig. 2).

The rate of development of hearing loss following cisplatin initiation was assessed using Kaplan-Meier survival analysis. The rate at which patients developed hearing loss differed significantly depending on the anatomical location of the tumour (log rank test, $p=0.022$ ). Patients with head and neck cancers or lymphoma developed hearing loss faster than the rest of the patients (Fig. 3). The odds of developing cisplatin-induced hearing loss were elevated for patients with head and neck tumours and lymphoma (logistic regression, odds ratio (OR) 1.824; $p=0.0465$ and $\mathrm{OR} 2.250$; $p=0.0563$, respectively), and significantly reduced for patients with reproductive cancers (OR $0.111 ; p=0.0371$ ). Notably, only 1 of the 10 patients with reproductive cancer developed hearing loss. The rate of hearing loss did not differ significantly according to gender (log rank test, $p=0.515$ ), age at initiation of treatment (log rank test, $p=0.490$ ) or severity of hearing loss (log rank test, $p=0.450$ ).

\section{Discussion}

Ototoxicity has been recognised as an adverse effect of cisplatin treatment for almost three decades ${ }^{[14]}$ yet because of its high cure rate ${ }^{[1]}$ it is administered in the first-line treatment of many cancer types. Furthermore, cisplatin has been shown to significantly increase the lifespan of patients, ${ }^{[15]}$ and the benefit of chemotherapeutic treatment often outweighs the risk of ototoxicity. Sustaining the patient's quality of life both during and after treatment is therefore of the utmost importance.

This is the first study that provides a quantitative analysis of cisplatin-induced ototoxicity in an adult SA population. With an incidence of $55.1 \%$, this report of cisplatininduced hearing loss illustrates that SA adult patients may be at higher risk than their European and South American counterparts, for whom incidence rates of $23-50 \%$ have been reported. ${ }^{[4-6]}$ We suspect that this trend would be similar for many other hospitals around SA. Since not all patients receive audiological monitoring during high-dose cisplatin treatment and this study was only conducted at a single major Western Cape hospital, our findings may under-represent the incidence of cisplatin-induced ototoxicity.

The increasing annual frequency of $300 \%$ for new cisplatin-receiving patients who were audiologically monitored during treatment between 2006 and 2010 can be attributed to improved awareness of the ototoxic effects of cisplatin on the part of SA oncologists. In 2009, it was reported that only half of SA oncologists referred patients receiving chemotherapy for audiological monitoring during treatment, even though $90 \%$ of them were aware that the drugs cause physical damage to the auditory system. ${ }^{[16]}$ In this cohort, a minimal number $(n=3)$ of patients experienced debilitating hearing loss (i.e. grades 3 and 4) when cisplatin was substituted for the less ototoxic carboplatin when hearing loss in the high frequencies was detected. This is testament to the benefits of the regular audiometric monitoring that these patients received during treatment.

Cisplatin-induced ototoxicity has been shown to be dependent on age at initiation of treatment, paediatric ( $<5$ years) and elderly patients ( $>40$ years) displaying increased susceptibility. ${ }^{\left[\sigma_{117]}\right.}$ In our study, patient ages ranged between 14 and 75 years; however, there was no significant difference in the age distribution for patients who did and did not experience ototoxicity. Patient gender was not identified as a risk factor for cisplatininduced ototoxicity until recently, when 


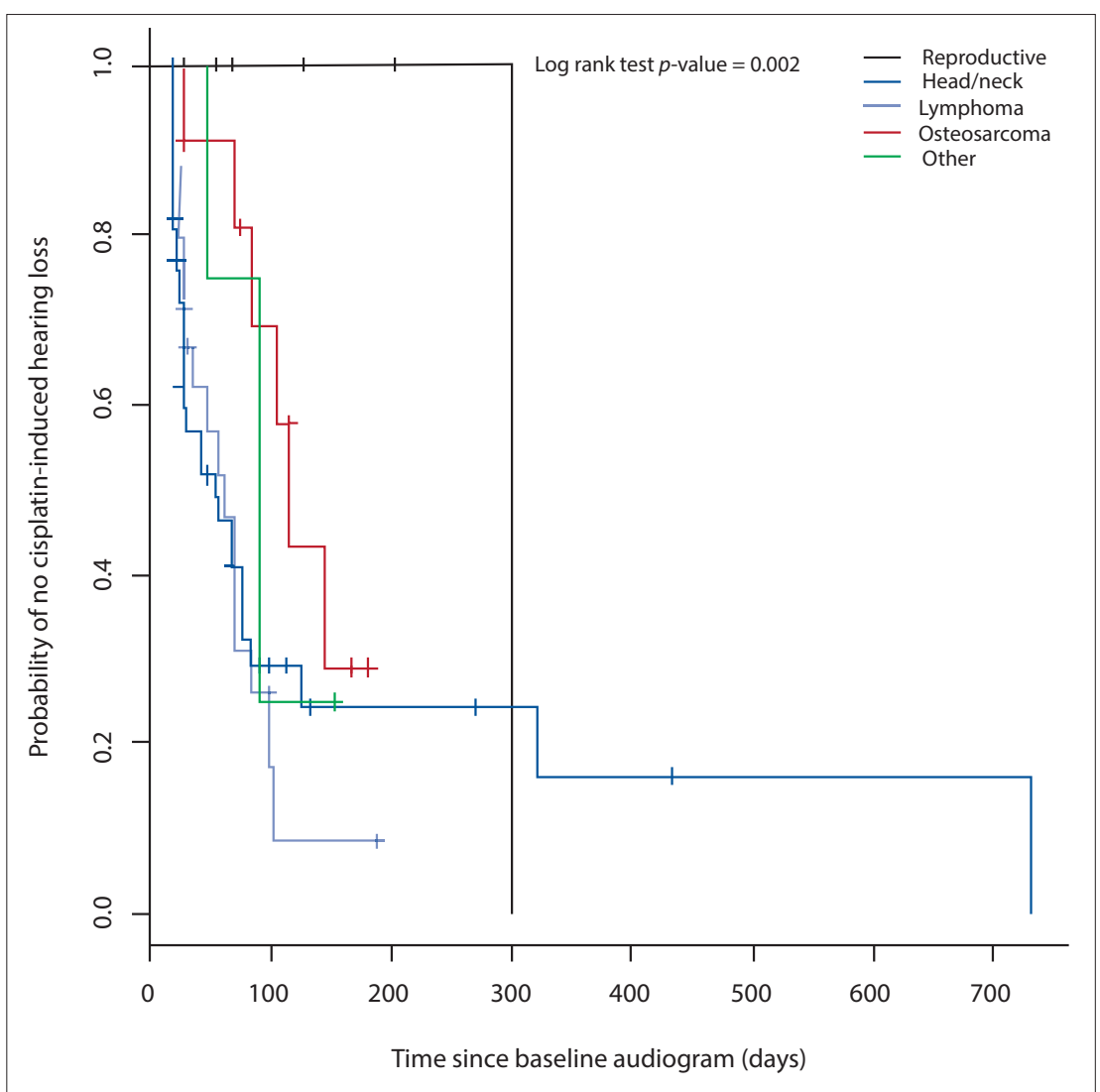

Fig. 3. Kaplan-Meier estimates of cisplatin-induced hearing loss-free survival based on anatomical site of cancer. Head/neck cancer and lymphoma patients developed hearing loss at a faster rate than the other cancer groups investigated.

Yancey et al. ${ }^{[9]}$ reported that male paediatric patients were four times more likely to experience ototoxicity than females. We found no association between gender and the development of significant hearing loss.

Cumulative dosages of cisplatin have been identified as the most important predictor of cisplatin-induced ototoxicity, particularly cumulative doses above $400 \mathrm{mg} / \mathrm{m}^{2} .^{[6,9,10]}$ In a recent study investigating a cohort of Japanese head and neck cancer patients, the threshold cumulative dosage of cisplatin was $200 \mathrm{mg} / \mathrm{m}^{2}$, above which average hearing thresholds were significantly reduced in these patients. ${ }^{[1]}$ Our data indicate that SA patients who received higher cumulative cisplatin dosages during chemotherapy were more likely to develop significant hearing loss, with median cumulative dosages of $180.70 \mathrm{mg} / \mathrm{m}^{2}$ and $236.84 \mathrm{mg} / \mathrm{m}^{2}$ for the ototoxicity-free and ototoxicity groups, respectively. Our findings therefore suggest that cumulative cisplatin dosage may be the most important predictor of ototoxcity in this population.

The risk of developing cisplatin-induced hearing loss was significantly reduced in patients with reproductive cancers. These patients receive each cycle of cisplatin treatment intravenously over a period of
3 - 5 days, which may account for the low incidence of ototoxicity in this group. The odds of developing cisplatin-induced hearing loss were elevated in head and neck cancer and lymphoma patients, and hearing loss occurred at a faster rate than in the rest of the patients investigated. Treatment for head and neck carcinomas and lymphomas usually involves surgery, chemotherapy and cranial irradiation, either singly or in combination. Although cranial irradiation was not investigated in this cohort, it has been identified as a risk factor for the development of ototoxicity. ${ }^{[18,19]}$ We hypothesise that the high incidence of hearing loss in these groups may be attributed to the use of cranial irradiation during treatment.

Other previously identified risk factors for cisplatin-induced ototoxicity, including previous exposure to ototoxic agents such as the aminoglycoside antibiotics and tuberculosis treatment, were not recorded. Previous noise exposure and renal dysfunction $^{[18,19]}$ were not investigated in this study owing to lack of clinical data.

The consequences of hearing loss can be debilitating and may limit the contribution of sufferers to society, adding to the economic burden on SA. Our data highlight the importance of regular audiological monitoring of patients receiving high-dose cisplatin treatment. Adequate knowledge of the complication of ototoxicity on the part of clinicians may facilitate the prevention or amelioration of further ototoxic damage through administration of otoprotective agents, or a change of chemotherapeutic drugs. Further research should aim to understand other risk factors, particularly genetic predictors of cisplatin-induced ototoxicity in the uniquely diverse SA population.

References

1. McKeage MJ. Comparative adverse effect profiles of platinum drugs. Drug Saf 1995;13(4):228-244. [http://dx.doi. org/10.2165/00002018-199513040-00003]

2. Siddik ZH. Cisplatin: Mode of cytotoxic action and molecular basis of resistance. Oncogene 2003;22(47):7265-7279. [http:// dx.doi.org/10.1038/sj.onc.1206933]

3. Buhrer C, Weinel P, Sauter S, Reiter A, Riehm H, Laszig R. Acute onset deafness in a 4-year old girl after a single infusion of cisplatinum. Pediatr Hematol Oncol 1990;7(2):145-148. [http:// dx.doi.org/10.3109/08880019009033384]

4. Coradini PP, Cigana L, Selistre SG, Rosito LS, Brunetto AL. Ototoxicity from cisplatin therapy in childhood cancer. J Pediatr Hematol Oncol 2007;29(6):355-360. [http://dx.doi.org/10.1097/ MPH.0b013e318059c220]

5. Knight KR, Kraemer DF, Neuwelt EA. Ototoxicity in children receiving platinum chemotherapy: Underestimating a commonly occurring toxicity that may influence academic and social development. J Clin Oncol 2005;23(34):8588-8596 [http://dx.doi.org/10.1200/JCO.2004.00.5355]

6. Li Y, Womer RB, Silber JH. Predicting cisplatin ototoxicity in children: The influence of age and the cumulative dose. Eur $\mathrm{J}$ Cancer 2004;40(16):2445-2451. [http://dx.doi.org/10.1016/j.ejca.2003.08.009]

7. Chang KW, Chinosornvatana N. Practical grading system for evaluating cisplatin ototoxicity in children. J Clin Oncol 2010;28(10):1788-1795. [http://dx.doi.org/10.1200/JCO.2009.24.4228]

8. Rybak LP, Whitworth CA, Mukherjea D, Ramkumar V Mechanisms of cisplatin-induced ototoxicity and prevention. Hear Res 2007;226(1-2):157-167. [http://dx.doi.org/10.1016/j. heares.2006.09.015]

9. Yancey A, Harris MS, Egbelakin A, Gilbert J, Pisoni DB Renbarger J. Risk factors for cisplatin-associated ototoxicity in paediatric oncology patients. Pediatr Blood Cancer 2012;59(1):144-148. [http://dx.doi.org/10.1002/pbc.24138]

10. Bokemeyer C, Berger CC, Haertmann JT, et al. Analysis of risk factors for cisplatin-induced ototoxicity in patients with testicular cancer. Br J Cancer 1998;77(8):1355-1362. [http:// dx.doi.org/10.1038/bjc.1998.226]

11. Eiamprapai P, Yamamoto N, Hiraumi H, et al. Effect of cisplatin on distortion product otoacoustic emissions in Japanes patients. Laryngoscope 2012:122(6):1392-1396. [http.//dx.doi patients. Laryngoscope
org/10.1002/lary.23336]

12. Campbell KC, Meech RP, Klemens JJ, et al. Prevention of

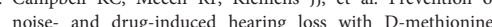
noise- and drug-induced hear 2007:226(1-2):92-103. [http//dx doi.org/10.1016/j. Hear Res 2007,2261

13. American Speech-Language-Hearing Association. Guidelines American Speech-Language-Hearing Association. Guidelines for the andologina receiving cochleotoxic drag therapy. ASHA Protice Policy 1994

14. Phtp://dx.doi.org/10.1044/policy.GL1994-00003]
(iel Meyer D, Perlia CP, Wolfe VI. Effects of cisdiamminedichloroplatinum (NSC-1 19875) on hearing function in man. Cancer Chemother Rep 1974;58(6):871-875.

15. Stewart BW, Kleihues P. World Health Organization International Agency for Research on Cancer. World Cancer Report. Lyon: IARC Press, 2003.

16. Andrade V, Khoza-Shangase K, Hajat F. Perceptions of oncologists at two state hospitals in Gauteng regarding the ototoxic effects of cancer chemotherapy: A pilot study. African Journal of Pharmacy and Pharmacology 2009;3(6):307-318.

17. Helson L, Okonkwo E, Anton L, Cvitkovic E. cis-Platinum ototoxicity. Clin Toxicol 1978;13(4):469-478. [http://dx.doi. org $/ 10.3109 / 15566$

18. Low WK, Toh ST, Wee J, Fook-Chong SM, Wang DY. Sensorineural hearing loss after radiotherapy and chemoradiotherapy: A single, blinded, randomized study. J Clin Oncol 2006;24(12):1904-1909. [http://dx.doi.org/10.1200/]CO.2005.05.0096]

19. Rednam S, Scheurer ME, Adesina A, Lau CC, Okcu MF. Glutathione S-transferase P1 single nucleotide polymorphism predicts permanent ototoxicity in children with medulloblastoma. Pediatr Blood Cancer 2013;60(4):593-598 [http://dx.doi.org/10.1002/pbc.24366]

Accepted 8 October 2013. 